\title{
Safety of Follitropin Alfa/Lutropin Alfa for Stimulation of Follicular Development
}

\author{
Nadezda Abramova ${ }^{1}\left(\mathbb{0} \cdot\right.$ Julie Hubbard $^{2} \cdot$ Joan Schertz $^{2} \cdot$ Emilia Richter $^{1}$
}

Published online: 19 October 2018

(c) The Author(s) 2018

\begin{abstract}
Introduction Recombinant human luteinizing hormone (r-hLH) is used in a fixed-ratio combination with recombinant human follicle-stimulating hormone (r-hFSH) for the stimulation of follicular development.

Objective The objective of this article was to conduct a review of safety data to evaluate the risks of $\mathrm{r}-\mathrm{hFSH} / \mathrm{r}-\mathrm{hLH}$ treatment. Methods Data were retrieved from the Global Safety Database (Merck KGaA, Darmstadt, Germany) including reports from healthcare professionals, patients, health authorities, clinical trials, non-interventional studies, and the literature. Reports of important risks (identified and potential) as per the risk management plan applicable at the time of data retrieval were obtained up to December 2017. The estimated patient exposure to r-hFSH/r-hLH in the post-marketing setting was 427,012 treatment cycles. Nine hundred patients received r-hFSH/r-hLH during company-sponsored clinical trials (pre- and post-marketing). Results We identified 72 case reports describing important risks related to r-hFSH/r-hLH use, including 46 cases of ovarian hyperstimulation syndrome (10.8 per 100,000 treatment cycles) and 24 of hypersensitivity reaction (5.6 per 100,000 treatment cycles). No thromboembolic events were reported. One congenital anomaly, not suspected to be related to r-hFSH/r-hLH use, was reported during a clinical trial; the event was resolved by corrective surgery. Two fatal cases were identified; one case of recurrent malignant melanoma (suspected to be related to $\mathrm{r}-\mathrm{hFSH} / \mathrm{r}-\mathrm{hLH}$ use) and one case resulting from complications of ovarian hyperstimulation syndrome.

Conclusion Cumulative reporting rates of important identified and potential risks of r-hFSH/r-hLH during a 10-year surveillance period demonstrate the benefit-risk balance is positive. This post-marketing surveillance and continued surveillance of safety events should provide reassurance about the use of r-hFSH/r-hLH in clinical practice.
\end{abstract}

Electronic supplementary material The online version of this article (https://doi.org/10.1007/s40264-018-0742-3) contains supplementary material, which is available to authorized users.

EMD Serono Research and Development Institute, Billerica, MA, USA, is a business of Merck KGaA, Darmstadt, Germany.

Nadezda Abramova

nadezda.abramova@merckgroup.com

1 Merck KGaA, Frankfurter Strasse 250, 64293 Darmstadt, Germany

2 EMD Serono Research and Development Institute, Billerica, MA, USA 


\section{Key Points}

Reports of important risks (identified and potential) for a fixed-ratio combination of recombinant human folliclestimulating hormone/recombinant human luteinizing hormone (r-hFSH/r-hLH) for the stimulation of follicular development in adult women with severe luteinizing hormone and follicle-stimulating hormone deficiency as per the risk management plan applicable at the time of data retrieval were obtained from the Global Safety Database maintained by Merck KGaA, Darmstadt, Germany

Data were included for the period up to December 2017, during which time the estimated patient exposure to r-hFSH/r-hLH in the post-marketing setting was 427,012 treatment cycles; 900 patients received $\mathrm{r}-\mathrm{hFSH} / \mathrm{r}-\mathrm{hLH}$ during Merck KGaA-sponsored clinical trials (pre- and post-marketing)

Cumulative reporting rates of important identified and potential risks of r-hFSH/r-hLH during a 10-year surveillance period demonstrate that the established benefitrisk balance is positive

\section{Introduction}

Recombinant human luteinizing hormone (r-hLH) is indicated in combination with recombinant human folliclestimulating hormone (r-hFSH) for the stimulation of follicular development in adult women with severe luteinizing hormone (LH) and follicle-stimulating hormone (FSH) deficiency [1]. Recombinant-human luteinizing hormone binds to $\mathrm{LH}$ receptors of ovarian theca and granulosa cells and stimulates ovarian theca cells to secrete androgens, which are then used as a substrate for the production of estradiol by the aromatase enzyme in granulosa cells. Estradiol, in turn, supports FSH-induced follicular development [1].

Treatment with r-hLH, initiated at a dose of 75 IU daily in combination with 75-150 IU r-hFSH, is indicated for the stimulation of follicular development in adult women with severe LH and FSH deficiency; these doses are subsequently individualized according to the patient's response to treatment [1]. In an effort to reduce the number of individual injections required and increase patient convenience, a fixed-ratio $2: 1$ combination of $\mathrm{r}-\mathrm{hFSH} / \mathrm{r}-\mathrm{hLH}$ [follitropin alfa/lutropin alfa (150:75 IU); Pergoveris ${ }^{\circledR}$; Merck KGaA, Darmstadt, Germany] was introduced in Europe in 2007, and is currently authorized in 92 countries [2]. Until recently, r-hFSH/r-hLH was only available as a freeze-dried powder, requiring reconstitution prior to injection; however, a liquid formulation of r-hFSH/r-hLH has been developed, and was granted marketing authorization by the European Medicines Agency on 8 May, 2017 [3]. This new formulation enables patients to administer $\mathrm{r}-\mathrm{hFSH} / \mathrm{r}-\mathrm{hLH}$ with a prefilled pen injector similar to the one currently used for GONAL- $\mathrm{f}^{\circledR}$ (r-hFSH; follitropin alfa; Merck KGaA), further increasing patient convenience by reducing the number of preparation steps required for an injection, as well as reducing the probability of dosing errors [4].

In most countries, $\mathrm{r}-\mathrm{hFSH} / \mathrm{r}-\mathrm{hLH}$ is indicated for the stimulation of follicular development in adult women with severe LH and FSH deficiency [2, 5]. It is also indicated for use in patients with sub-optimal response after controlled ovarian stimulation undergoing assisted reproductive technologies (ART) in Mexico, Russia, and Ukraine. The use of r-hLH (either as a fixed-ratio combination of r-hFSH/r-hLH or as a separate preparation in combination with $\mathrm{FSH}$ ) in women with different indications (including severe LH and FSH deficiency and ART) has previously been published [6-8]. Recombinant-human luteinizing hormone (both as r-hLH and a fixed-ratio combination of r-hFSH/r-hLH) has been used for controlled ovarian stimulation in normogonadotropic women and as part of ART treatments in women of advanced maternal age ( $\geq 35$ years), as well as in patients with poor ovarian reserve [6]. These uses have also been observed in post-marketing surveillance by Merck KGaA.

It is important that post-marketing safety data are closely monitored and available in the public domain to enable clinicians and patients to evaluate any potential treatment risks. This article presents the reporting rates of cases that include important risks for the fixed-ratio combination of r-hFSH/ r-hLH in the Global Safety Database maintained by Merck $\mathrm{KGaA}$. This should enable the assessment of the benefit-risk balance of this drug in routine clinical practice.

\section{Methods}

The Global Safety Database (Adverse Reaction Information System global, ARISg; ArisGlobal, CT, USA) of Merck $\mathrm{KGaA}$ comprises data for adverse events from clinical trials, observational/non-interventional studies, market research, patient or healthcare provider surveys, patients-support programs sponsored by Merck KGaA, its affiliates or investigators, spontaneous post-marketing reports, and surveillance of the published literature. We performed searches of ARISg for the fixed-ratio combination of r-hFSH/r-hLH (Pergoveris ${ }^{\circledR}$; Merck KGaA) and included data up to December 2017.

\subsection{Events Considered of Interest}

Every drug that receives marketing authorization requires a risk management plan (RMP), which includes information 
on the safety profile of the drug, how risks will be prevented or minimized, plans for further studies related to the safety and the efficacy of the drug, and details of how the effectiveness of risk-minimization measures will be evaluated. Important risks are those characterized and confirmed to have an impact on the benefit-risk balance of the drug or have implications for public health [9]. Important risks for $\mathrm{r}-\mathrm{hFSH} / \mathrm{r}-\mathrm{hLH}$ based on the RMP applicable at the time of data retrieval can be classified as identified or potential:

- Important identified risks, for which there is adequate evidence of an association with $\mathrm{r}-\mathrm{hFSH} / \mathrm{r}-\mathrm{hLH}$ include ovarian hyperstimulation syndrome (OHSS; subdivided by severity, i.e., mild, moderate, or severe), thromboembolic events, usually in association with OHSS, and hypersensitivity reactions (allergic and pseudoallergic; subdivided by severity, i.e., mild, moderate, or severe).

- Important potential risks, for which there is suspicion of an association with r-hFSH/r-hLH but where this association has not been confirmed, include specific cancers (including breast, ovarian, uterine, and malignant melanoma) and congenital anomalies [9]. Important potential risks are areas closely monitored by routine pharmacovigilance practice [10].

\subsection{Search Strategy}

We searched the Global Safety Database for individual case safety reports (ICSRs) of important identified and potential risks that meet the criteria mentioned below.

- Post-marketing sources (excluding Merck KGaA-sponsored, post-marketing interventional clinical trials): cover reports from the post-marketing period available in the safety database including all ICSRs (irrespective of causality assessment by either the investigator or sponsor) from healthcare professionals, consumers (including cases from patient data collection systems, such as patient support programs), non-interventional clinical studies sponsored by Merck KGaA, its affiliates or predecessor companies, interventional studies not sponsored by Merck KGaA, the scientific literature, and regulatory authorities.

- Clinical trial (Merck KGaA-sponsored interventional clinical studies) sources: cover reports available in the safety database from any interventional clinical trial sponsored by Merck KGaA, its affiliates or predecessor companies, regardless of the causality assessment by either the investigator or sponsor.

Throughout this article, ICSRs are classified depending on the source as either post-marketing or clinical trial. Each case report could include more than one safety event, but signs and/or symptoms reported with the main diagnosis/ event were not recorded as separate events. The database search was conducted using the Medical Dictionary for Regulatory Activities (MedDRA ${ }^{\circledR}$ ) Version 20.1 terms [11]. The search terms are listed in the Electronic Supplementary Material (ESM). The Global Safety Database was designed to monitor these events and includes OHSS, thromboembolic events, usually in association with OHSS, hypersensitivity reactions, specific cancers and congenital anomalies, as well as screening of the literature.

Literature searches were performed on a weekly basis to supplement the safety database with the relevant literature that reported important identified and potential risks. MEDLINE and EMBASE databases were searched comprehensively using various compilations of free text search terms (including active ingredients and respective brand names) and index terms (controlled terms from the database thesauri). The inclusion of additional searches from publicly available safety databases (e.g., VigiBase) was considered to likely produce duplicate results and therefore was not included in this analysis. Furthermore, as r-hFSH/ $\mathrm{r}$-hLH does not have marketing authorization in USA and not all countries participate in the Vigibase initiative, the Global Safety Database from Merck KGaA was considered the most comprehensive repository of ICSRs relating to r-hFSH/r-hLH.

\subsection{Analysis}

\subsubsection{Causality}

Each individual, important, identified and potential risk was evaluated separately. The ICSRs pertaining to each important risk were retrieved from the database, according to the pre-defined search strategy outlined in Sect. 2.2. All initial spontaneous case reports received from the reporter are considered to be suspected to be related to the product, unless the reporters specifically state that they believe the events to be unrelated or that a causal relationship can be excluded [12]. A secondary causality assessment is conducted by Merck KGaA and the aggregate result of both assessments is recorded in the Global Safety Database. In all cases, the causality of each event was assessed and classified, as follows [9]:

- Suspected: the event is reasonably related to the medicinal product. The event can medically (pharmacologically or clinically) be attributed to the reported medicinal product. "Suspected" was selected in the database as the reporter's assessment of causality, if reported as "probable", "possible", "unlikely", or "unknown". 
- Not suspected: the event is not reasonably related to the medicinal product. The event could not medically (pharmacologically or clinically) be attributed to the reported medicinal product. "Not suspected" was selected in the database as the reporter's assessment of causality, if reported as "not related".

\subsubsection{Seriousness}

Safety events can be classified as serious or non-serious. A serious event was defined as a medical occurrence that [13]:

- resulted in death;

- was life threatening;

- resulted in hospitalization (initial or prolonged);

- resulted in disability or permanent damage;

- was a congenital anomaly/birth defect;

- was a medically important event or reaction.

The events that do not fulfill the above-mentioned criteria were considered to be non-serious.

\subsubsection{Reporting Rates}

The reporting rate for each safety concern was calculated by dividing the number of cases reported by the estimated number of treatment cycles that occurred since the product's international birth date (25 June, 2007). Ideally, the reporting rate should be based on the number of patients exposed to r-hFSH/r-hLH; however, owing to the available data (number of units sold) and the fact that in clinical practice patients may receive multiple cycles, the number of patients who received r-hFSH/r-hLH cannot be accurately estimated. As such, the number of treatment cycles was selected as the denominator as it is the most clinically relevant data that could be determined. The number of treatment cycles was estimated based on the number of vials and prefilled $\mathrm{r}-\mathrm{hFSH} / \mathrm{r}-\mathrm{hLH}$ pens sold; it was assumed that one vial of r-hFSH/r-hLH (150 IU/75 IU) is administered per day per patient and the average duration of one treatment cycle is 12 days. Cartridges for prefilled $\mathrm{r}-\mathrm{hFSH} / \mathrm{r}-\mathrm{hLH}$ pens were converted to vials and included in the estimation. Statistical analyses are descriptive; quantitative analyses of data were not feasible as the database includes a population of uncertain size.

\section{Results}

The total post-marketing patient exposure to r-hFSH/r-hLH from its international birth date to 24 December, 2017 was estimated to be 427,012 treatment cycles based on sales data. In addition, 900 patients received r-hFSH/r-hLH during Merck KGaA-sponsored pre- and post-marketing clinical trials. During this time, 72 ICSRs of events considered important identified and potential risks relating to the use of $\mathrm{r}-\mathrm{hFSH} / \mathrm{r}-\mathrm{hLH}$ were received (Table 1); among these, 68 were reported during the post-marketing period, and four were reported during clinical trials.

\subsection{Important Identified Risks}

\subsubsection{Ovarian Hyperstimulation Syndrome}

In total, there were 46 ICSRs of OHSS (Tables 1 and 2), three of which were reported during Merck KGaA-sponsored clinical trials. The remaining 43 ICSRs of OHSS were reported during the post-marketing period; 32 were considered as serious and 14 as non-serious. All cases were suspected to be related to the use of r-hFSH/r-hLH. The outcome was known for 26 of the 46 cases and, in the majority (21 of 26), the events were resolved and the patients recovered (Table 2). The cumulative reporting rate of all degrees of severity of OHSS with $\mathrm{r}-\mathrm{hFSH} / \mathrm{r}-\mathrm{hLH}$ was estimated at 10.8 cases per 100,000 treatment cycles $(0.011 \%)$.

One case of a fatal outcome resulting from a number of OHSS-related complications was reported. The patient was a 26-year-old woman who initiated treatment with r-hFSH/ r-hLH (150 IU/75 IU daily for 10 days), cetrorelix acetate (Cetrotide $^{\circledR}$; Merck KGaA; $0.25 \mathrm{mg}$ daily for 4 days), and recombinant human chorionic gonadotropin (Ovitrelle ${ }^{\circledR}$;

Table 1 Reports of important risks relating to the use of recombinant human luteinizing hormone/recombinant human follicle-stimulating hormone

\begin{tabular}{lcl}
\hline Important risk & $\begin{array}{l}\text { Cumula- } \\
\text { tive no. of } \\
\text { ICSRs }^{\mathrm{a}}\end{array}$ & $\begin{array}{l}\text { Cumulative reporting rate } \\
\text { (per 100,000 treatment } \\
\text { cycles) }^{\mathrm{b}}\end{array}$ \\
\hline OHSS & $46^{\mathrm{c}}$ & 10.8 \\
Thromboembolic events & 0 & - \\
$\begin{array}{l}\text { Hypersensitivity } \\
\begin{array}{l}\text { Breast, ovarian, and } \\
\text { uterine cancer }\end{array}\end{array}$ & 24 & 5.6 \\
$\begin{array}{l}\text { Malignant melanoma } \\
\text { Congenital anomaly }\end{array}$ & 1 & - \\
\hline
\end{tabular}

ICSRs individual case safety reports, OHSS ovarian hyperstimulation syndrome

${ }^{a}$ Cumulative number includes ICSRs from clinical trials and postmarketing (including spontaneous post-marketing reporting, interventional studies not sponsored by Merck KGaA, observational/ non-interventional studies and market research, patient or healthcare provider surveys or patients-support programs)

${ }^{\mathbf{b}}$ Calculated based on the reported cumulative total exposure of 427,012 treatment cycles

${ }^{\mathrm{c}}$ Includes three cases reported during clinical trials

${ }^{\mathrm{d}}$ Uterine cancers include endometrial and cervical

${ }^{\mathrm{e}}$ Includes one case reported during a clinical trial 


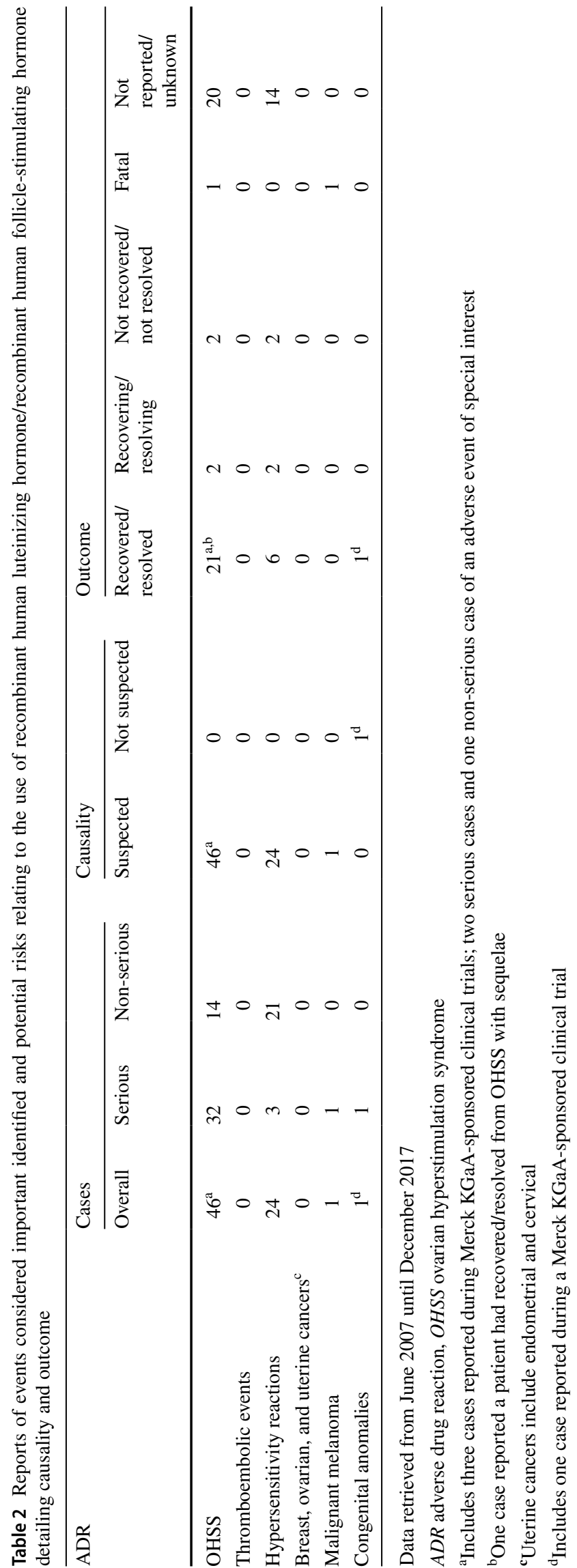

Merck KGaA; once) for controlled ovarian stimulation on 24 January, 2014. The patient did not have previous exposure to ovarian stimulation and infertility was attributed to male factor. The patient's relevant medical history included low estradiol levels ( $29 \mathrm{pg} / \mathrm{mL})$, a thin endometrium $(4 \mathrm{~mm})$, and normal FSH and LH levels $(7.2 \mathrm{mIU} / \mathrm{mL}$ and $5.6 \mathrm{mIU} / \mathrm{mL}$, respectively). Concomitant medications included acetylsalicylic acid, nadroparin calcium, cholecalciferol, calcium carbonate, $8 \%$ progesterone gel (Crinone ${ }^{\circledR}$; Merck KGaA), and dydrogesterone.

The patient developed mild OHSS following oocyte retrieval and embryo transfer, clinically manifested by fluid in the abdominal cavity that lasted for 3 days, with abdominal pain, swelling, distension, and ascites; approximately $2 \mathrm{~L}$ of accumulated fluid was removed from the abdominal cavity. Ovarian hyperstimulation syndrome was assessed as moderate at the beginning and the patient received outpatient treatment, but was later hospitalized for observation. In the hospital, additional fluid was removed from the abdominal cavity and pleural effusion drainage was also required. Pregnancy was confirmed by ultrasound but the patient died the following day. The subsequent autopsy revealed that death was due to acute respiratory distress syndrome caused by a left-sided hemothorax. The reactions that led to the fatal outcome (OHSS, acute respiratory distress syndrome, and left-sided hemothorax) were assessed by Merck KGaA, as suspected to be related to $\mathrm{r}-\mathrm{hFSH} / \mathrm{r}-\mathrm{hLH}$, in the absence of sufficient information to fully assess the case.

\subsubsection{Thromboembolic Events, Usually Associated with Ovarian Hyperstimulation Syndrome}

No ICSRs of thromboembolic events were retrieved from the Global Safety Database. Despite this, thromboembolic events are still considered by Merck KGaA as an important identified risk, given that they are complications typically associated with severe OHSS.

\subsubsection{Hypersensitivity Reactions}

There were a total of 24 ICSRs of hypersensitivity reactions (Tables 1 and 2), all of which were reported during the post-marketing period. In all cases, a causal association between $\mathrm{r}-\mathrm{hFSH} / \mathrm{r}-\mathrm{hLH}$ and the reported event could not be determined and 21 of 24 cases were considered non-serious. The outcome was known for 10 of the 24 cases, with the patient reported to have recovered and the event resolved in six of these cases. None of the cases was life threatening or fatal. The post-marketing cumulative reporting rate of hypersensitivity reactions was estimated at 5.6 per 100,000 treatment cycles $(0.006 \%)$. 


\subsection{Important Potential Risks}

\subsubsection{Specific Cancers (Including Breast, Ovarian, Uterine, and Melanoma)}

There were no ICSRs of breast, ovarian, or uterine cancer (including endometrial and cervical) retrieved from the Global Safety Database. However, there was one ICSR of malignant melanoma (estimated cumulative reporting rate of 0.2 per 100,000 treatment cycles; $0.0002 \%$ ) reported during the post-marketing period. This case was considered serious with a fatal outcome.

The patient was a 41-year-old woman who had a recurrence of malignant melanoma with metastases whilst receiving treatment with $\mathrm{r}-\mathrm{hFSH} / \mathrm{r}-\mathrm{hLH}$. Infertility was attributed to endometriosis and the patient's age. The patient had previously undergone three in-vitro fertilization treatment cycles and two ovulation stimulation cycles with follitropin alpha $\left(\right.$ GONAL- $\left.\mathrm{f}^{\circledR}\right)$ and other unknown products, and had a prior history of malignant melanoma on the right arm, which had been treated with surgery and subsequent radiotherapy. The type, stage, and thickness of the initial melanoma was not reported. Following treatment for melanoma, the patient was considered cured and melanoma did not reoccur in the 5 years prior to the fatal incident. The patient had received three ovarian stimulation cycles with r-hFSH/r-hLH (three ampoules per day; cycle duration: 10, 8, and 7 days), each followed by administration of recombinant human chorionic gonadotropin (Ovitrelle ${ }^{\circledR}$; once per cycle) and 8\% progesterone gel (Crinone ${ }^{\circledR}$; once per day). During the last stimulation cycle with $\mathrm{r}-\mathrm{hFSH} / \mathrm{r}-\mathrm{hLH}$, malignant melanoma recurred and metastasized, which led to discontinuation of the stimulation cycle by the patient. The outcome from the malignant melanoma was fatal. The events of recurrent malignant melanoma and metastasis were determined by the reporter as not assessable, given the lack of additional information. As such, Merck KGaA determined this case to be a suspected adverse drug reaction, as a possible causal link could not be ruled out. Since this case was reported in 2010, there have been no other cases of malignant melanoma.

\subsubsection{Congenital Anomalies}

One ICSR of a congenital anomaly was reported during a clinical trial (estimated cumulative reporting rate of 0.2 per 100,000 treatment cycles; $0.0002 \%$ ). This was considered a serious case in which a 9-month-old infant was diagnosed with atrial and ventricular septal defects, following fetal exposure to r-hFSH/r-hLH in utero. These defects were resolved by corrective surgery. The events of atrial and ventricular septal defects were assessed as not suspected to be related to r-hFSH/r-hLH. No congenital anomalies were reported during the post-marketing period.

\section{Discussion}

This article reports safety data with a fixed-ratio combination of r-hFSH/r-hLH; 72 ICSRs were retrieved from the safety database from different sources, including four cases from Merck KGaA-sponsored clinical trials. This included approximately 427,012 treatment cycles during the post-marketing period and 900 patients during Merck KGaA-sponsored interventional clinical trials.

The cases discussed here are related to important identified and potential risks. According to the RMP for $\mathrm{r}-\mathrm{hFSH} / \mathrm{r}-\mathrm{hLH}$ applicable at the time of the data retrieval, important identified risks include OHSS, thromboembolic events, usually associated with OHSS, and hypersensitivity reactions, and important potential risks include specific cancers and congenital anomalies.

The most frequently reported adverse event suspected to be related to the use of r-hFSH/r-hLH was OHSS (46 cases). Ovarian hyperstimulation syndrome is a serious complication associated with the use of gonadotropins for ovarian stimulation [14] and can present with various degrees of severity. Mild manifestations of OHSS may include abdominal pain, abdominal discomfort and distension, and enlarged ovaries; patients with moderate OHSS may additionally present with nausea, vomiting, ascites, and marked ovarian enlargement. Because OHSS is a well-known risk that is well diagnosed and managed in clinical practice, mild or moderate OHSS usually resolves spontaneously [1, 15], these cases often remain unreported, which might partly explain the low reporting rate of cases of OHSS observed in the post-marketing period in the safety database. In addition, under-reporting of safety concerns to external agencies has been observed to be a common occurrence because of time constraints and duplication of documentation and is likely to be a contributing factor in the low reporting rate of OHSS [16]. The low reporting rate of OHSS in patients treated with $\mathrm{r}-\mathrm{hFSH} / \mathrm{r}-\mathrm{hLH}$ could also be partially owing to the fact that $\mathrm{r}-\mathrm{hFSH} / \mathrm{r}-\mathrm{hLH}$ is indicated for stimulation of follicular development in adult women with severe LH and FSH deficiency who have been demonstrated to have the lowest risk of OHSS [17].

The incidence of OHSS in the in-vitro fertilization population is difficult to estimate because of the lack of an internationally agreed-upon classification system [15]. In 2010, the European IVF-Monitoring Consortium (25 countries) found that OHSS was reported at a prevalence of $0.3 \%$ of all stimulated cycles in the countries reporting the data [18]. However, the incidence of OHSS varies across different types of fertility treatment, given that different clinical situations may not be comparable in terms of therapeutic goals and strategies [19]. In conventional 
in-vitro fertilization, mild OHSS has been observed in $20-23 \%$ of cycles, moderate OHSS in $3-6 \%$ of cycles, and severe OHSS in $0.1-2 \%$ of cycles [20]. Considering these data, the reporting rate of OHSS with $\mathrm{r}-\mathrm{hFSH} / \mathrm{r}-\mathrm{hLH}$ $(0.011 \%)$ during the post-marketing period was below the expected incidence of OHSS in women treated with exogenous gonadotropins.

Death as a result of OHSS is very rare; since the international birth date of r-hFSH/r-hLH, only one fatal case of OHSS was reported. The patient had a reported diagnosis of OHSS and the autopsy showed that death was due to acute respiratory distress syndrome caused by a left-sided hemothorax, which was unlikely to have been a direct consequence of OHSS and was more likely to be related to bleeding following drainage of a pleural effusion. In the real world, only three deaths as a result of OHSS in over 1 million treatment cycles were reported between 1984 and 2008 in the Netherlands (it is not known whether these deaths were attributed to gonadotropin treatment); none were reported in the UK between 2006 and 2008 (approximately 73,500 treatment cycles) or in Israel between 1987 and 1996 (approximately 119,000 treatment cycles) [15]. The low number of OHSS cases with a fatal outcome could be owing to the numerous methods that exist for preventing OHSS, including the common practice of dose individualization, the administration of a gonadotropin-releasing hormone agonist in place of human chorionic gonadotropin to induce final follicle/oocyte maturation in gonadotropin-releasing hormone antagonist cycles, and subsequent cryopreservation of embryos or cycle cancellations in an attempt to avoid ovarian hyperstimulation altogether [21]. Furthermore, patients are usually closely monitored following oocyte retrieval to ensure that OHSS is identified and treated as soon as it is diagnosed.

There were few reported hypersensitivity reactions suspected to be related to use of r-hFSH/r-hLH, and the majority of cases were non-serious, with no fatal or life-threatening cases. In addition, there were no reported cases of thromboembolic events.

In general, ovarian, endometrial, and breast cancers are associated with risk factors that are usually present in infertile patients, such as low parity, infertility, early age at menarche, and late age at menopause [22]. Early clinical trials suggested a possible increased risk for these cancers in women receiving fertility treatments, although this view has since changed as more data have become available [22-25]. There were no case reports of reproductive system cancers identified from the Global Safety Database, suggesting there is no increased risk for these events. Throughout the post-marketing period, only one case of recurrent malignant melanoma with $\mathrm{r}-\mathrm{hFSH} / \mathrm{r}-\mathrm{hLH}$ treatment was reported. This case was considered serious, as it had a fatal outcome. However, there were a number of confounding factors that might have contributed to this event, including the patient's prior medical history of malignant melanoma (the type, stage, and thickness of which are unknown) and subsequent treatment with radiotherapy, as patients with previous malignancies or a history of radiation therapy carry a significant risk for recurrence and metastases. This report was received in 2010 and since then, there have been no case reports of malignant melanoma. Despite this, Merck KGaA still considers it an important potential risk that is closely monitored.

One case of congenital anomaly was identified from an interventional clinical trial sponsored by Merck KGaA, but there was no suspected causal association between the use of $\mathrm{r}-\mathrm{hFSH} / \mathrm{r}-\mathrm{hLH}$ and the reported event. In the general population, the prevalence of congenital anomalies is approximately $2.7 \%$ of all births [26] and it has been reported that women who undergo ART are more likely to give birth to babies with congenital anomalies than women who conceive spontaneously $[27,28]$. The occurrence of congenital anomalies with $\mathrm{r}-\mathrm{hFSH} / \mathrm{r}-\mathrm{hLH}$ during the post-marketing surveillance period, including clinical trials $(0.0002 \%)$, fell below the expected frequency, suggesting that congenital anomalies are not suspected to be related to the use of $\mathrm{r}-\mathrm{hFSH} / \mathrm{r}-\mathrm{hLH}$, but these events are still closely monitored by Merck KGaA.

The analysis reported here includes safety data that were rigorously collected from clinical trials and mandatory postmarketing safety monitoring of $\mathrm{r}-\mathrm{hFSH} / \mathrm{r}-\mathrm{hLH}$. In the postmarketing setting, data are collected directly from healthcare professionals, health authorities, consumer reports, and also from the scientific literature. Therefore, the Global Safety Database of Merck KGaA represents one of the best comprehensive sources for safety data on this drug.

However, there are a number of limitations that should be considered when interpreting the results. First, this analysis was limited to specific ICSRs related to important identified and potential risks listed in the RMP for $\mathrm{r}-\mathrm{hFSH} / \mathrm{r}-\mathrm{hLH}$ applicable at the time of data retrieval. Second, the cases reported in the post-marketing setting (i.e., excluding those from clinical trials sponsored by Merck KGaA and its affiliates or investigators) were either submitted voluntarily or obtained from the literature, precluding statistical analyses because of the lack of reliable patient exposure data. In addition, owing to a less stringent reporting process than in a clinical trial environment, all relevant information relating to each case reported in the post-marketing period may not be available, including the indication for which the drug was used. Third, the number of treatment cycles has been estimated from sales data. However, because the duration of treatment is adjusted to the individual patient, it is difficult to determine the total exposure of $\mathrm{r}-\mathrm{hFSH} / \mathrm{r}-\mathrm{hLH}$ in patients. Finally, not all of the product sold for a treatment cycle may be used. As such, the reporting rates (based on estimated total exposure in the denominator) should only be considered as approximations. 


\section{Conclusion}

This review of safety data for approximately 427,000 treatment cycles with r-hFSH/r-hLH and 900 patients who participated in interventional studies sponsored by Merck KGaA demonstrates that the established benefit-risk balance of $\mathrm{r}-\mathrm{hFSH} / \mathrm{r}-\mathrm{hLH}$ is positive. This post-marketing surveillance and continued surveillance of safety events should provide reassurance to both physicians and patients about the use of $\mathrm{r}-\mathrm{hFSH} / \mathrm{r}-\mathrm{hLH}$ in clinical practice.

Acknowledgements The authors thank Samia Bouhired, Jacek Bulicz, and Thomas Bauknecht for their assistance in obtaining data for this manuscript and Erica Velthuis for contributing to critical discussions on this manuscript. They also thank Alexander Jones and Ricky Tsang of inScience Communications, Springer Healthcare, UK for providing medical writing support in the preparation of this manuscript. This support was funded by Merck KGaA, Darmstadt, Germany.

Author Contributions All authors contributed to the conception and design of the analysis, as well as interpretation of data and critical review of this manuscript. All authors approved the manuscript for submission to the journal.

\section{Compliance with Ethical Standards}

Funding This analysis was funded by Merck KGaA, Darmstadt, Germany.

Conflict of interest Nadezda Abramova and Emilia Richter are employees of Merck KGaA, Darmstadt, Germany. Julie Hubbard and Joan Schertz are employees of the EMD Serono Research and Development Institute, Billerica, MA, USA, a business of Merck KGaA, Darmstadt, Germany.

Open Access This article is distributed under the terms of the Creative Commons Attribution-NonCommercial 4.0 International License (http://creativecommons.org/licenses/by-nc/4.0/), which permits any noncommercial use, distribution, and reproduction in any medium, provided you give appropriate credit to the original author(s) and the source, provide a link to the Creative Commons license, and indicate if changes were made.

\section{References}

1. Luveris ${ }^{\circledR}$ : European summary of product characteristics. 2017. http://www.ema.europa.eu/docs/en_GB/document_library/ EPAR_-_Product_Information/human/000292/WC50004591 4.pdf. Accessed 5 Oct 2018.

2. Pergoveris ${ }^{\circledR}$ : European summary of product characteristics. 2017. http://www.ema.europa.eu/docs/en_GB/document_libra ry/EPAR_-_Product_Information/human/000714/WC50003998 3.pdf. Accessed 5 Oct 2018.

3. European Medicines Agency. Pergoveris ${ }^{\circledR}$ : EPAR. All authorized presentations. 2017. http://www.ema.europa.eu/docs/en_GB/ document_library/EPAR_-_All_Authorised_presentations/human /000714/WC500039985.pdf. Accessed 5 Oct 2018.
4. Landfeldt E, Jablonowska B, Norlander E, Persdotter-Eberg K, Thurin-Kjellberg A, Wramsby M, et al. Patient preferences for characteristics differentiating ovarian stimulation treatments. Hum Reprod. 2012;27(3):760-9.

5. Rinaldi L, Selman H. Profile of follitropin alpha/lutropin alpha combination for the stimulation of follicular development in women with severe luteinizing hormone and follicle-stimulating hormone deficiency. Int J Womens Health. 2016;8:169-79.

6. Lehert P, Kolibianakis EM, Venetis CA, Schertz J, Saunders $\mathrm{H}$, Arriagada $\mathrm{P}$, et al. Recombinant human follicle-stimulating hormone ( $\mathrm{r}-\mathrm{hFSH})$ plus recombinant luteinizing hormone versus r-hFSH alone for ovarian stimulation during assisted reproductive technology: systematic review and meta-analysis. Reprod Biol Endocrinol. 2014;20(12):17. https://doi. org/10.1186/1477-7827-12-17.

7. Buhler K, Naether OG, Bilger W. A large, multicentre, observational, post-marketing surveillance study of the 2:1 formulation of follitropin alfa and lutropin alfa in routine clinical practice for assisted reproductive technology. Reprod Biol Endocrinol. 2014;14(12):6. https://doi.org/10.1186/1477-7827-12-6.

8. Vuong TN, Phung HT, Ho MT. Recombinant follicle-stimulating hormone and recombinant luteinizing hormone versus recombinant follicle-stimulating hormone alone during GnRH antagonist ovarian stimulation in patients aged $\geq 35$ years: a randomized controlled trial. Hum Reprod. 2015;30(5):1188-95.

9. European Medicines Agency. Guideline on good pharmacovigilance practices (GVP); Annex I: definitions (Rev 4). 2017. http:// www.ema.europa.eu/docs/en_GB/document_library/Scientific _guideline/2013/05/WC500143294.pdf. Accessed 5 Oct 2018.

10. European Medicines Agency. Guidance on the format of the risk management plan (RMP) in the EU: in integrated format. 2017 [updated 30 March 2017]. http://www.ema.europa.eu/docs/ en_GB/document_library/Regulatory_and_procedural_guide line/2017/03/WC500224771.pdf. Accessed 5 Oct 2018.

11. Medical Dictionary for Regulatory Activities (MedDRA). 2017. https://www.meddra.org/. Accessed 5 Oct 2018.

12. European Medicines Agency. Guideline on good pharmacovigilance practices (GVP); Module VI: collection, management and submission of reports of suspected adverse reactions to medicinal products (Rev 2). 2017. http://www.ema.europa.eu/docs/ en_GB/document_library/Regulatory_and_procedural_guide line/2017/08/WC500232767.pdf. Accessed 5 Oct 2018.

13. International Conference on Harmonisation. Post-approval safety data management: definitions and standards for expedited reporting (E2D). 2003. https://www.ich.org/fileadmin/Public_Web_Site/ ICH_Products/Guidelines/Efficacy/E2D/Step4/E2D_Guideline. pdf. Accessed 5 Oct 2018.

14. Gibreel A, Bhattacharya S. Recombinant follitropin alfa/lutropin alfa in fertility treatment. Biologics. 2010;4:5-17.

15. Royal College of Obstetricians and Gynaecologists. The management of ovarian hyperstimulation syndrome. 2016. https://www. rcog.org.uk/globalassets/documents/guidelines/green-top-guide lines/gtg_5_ohss.pdf. Accessed 5 Oct 2018.

16. Hohl CM, Small SS, Peddie D, Badke K, Bailey C, Balka E. Why clinicians don't report adverse drug events: qualitative study. JMIR Public Health Surveill. 2018;4(1):e21.

17. Ashrafi M, Bahmanabadi A, Akhond MR, Arabipoor A. Predictive factors of early moderate/severe ovarian hyperstimulation syndrome in non-polycystic ovarian syndrome patients: a statistical model. Arch Gynecol Obstet. 2015;292(5):1145-52.

18. Kupka MS, Ferraretti AP, de Mouzon J, Erb K, D’Hooghe T, Castilla JA, et al. Assisted reproductive technology in Europe, 2010: results generated from European registers by ESHREdagger. Hum Reprod. 2014;29(10):2099-113. 
19. Delvigne A, Rozenberg S. Epidemiology and prevention of ovarian hyperstimulation syndrome (OHSS): a review. Hum Reprod Update. 2002;8(6):559-77.

20. Vloeberghs V, Peeraer K, Pexsters A, D'Hooghe T. Ovarian hyperstimulation syndrome and complications of ART. Best Pract Res Clin Obstet Gynaecol. 2009;23(5):691-709.

21. Mathur RS, Tan BK. British Fertility Society Policy and Practice Committee: prevention of ovarian hyperstimulation syndrome. Hum Fertil (Camb). 2014;17(4):257-68.

22. Cetin I, Cozzi V, Antonazzo P. Infertility as a cancer risk factor: a review. Placenta. 2008;29:169-77.

23. Rizzuto I, Behrens RF, Smith LA. Risk of ovarian cancer in women treated with ovarian stimulating drugs for infertility. Cochrane Database Syst Rev. 2013;8:CD008215.

24. van den Belt-Dusebout AW, Spaan M, Lambalk CB, Kortman M, Laven JS, van Santbrink EJ, et al. Ovarian stimulation for in vitro fertilization and long-term risk of breast cancer. JAMA. 2016;316(3):300-12.
25. Li LL, Zhou J, Qian XJ, Chen YD. Meta-analysis on the possible association between in vitro fertilization and cancer risk. Int $\mathrm{J}$ Gynecol Cancer. 2013;23(1):16-24.

26. Boyle B, Addor MC, Arriola L, Barisic I, Bianchi F, CsakySzunyogh M, et al. Estimating global burden of disease due to congenital anomaly: an analysis of European data. Arch Dis Child Fetal Neonatal Ed. 2018;103(1):F22-8.

27. Levi Setti PE, Moioli M, Smeraldi A, Cesaratto E, Menduni F, Livio S, et al. Obstetric outcome and incidence of congenital anomalies in 2351 IVF/ICSI babies. J Assist Reprod Genet. 2016;33(6):711-7.

28. Heisey AS, Bell EM, Herdt-Losavio ML, Druschel C. Surveillance of congenital malformations in infants conceived through assisted reproductive technology or other fertility treatments. Birth Defects Res A Clin Mol Teratol. 2015;103(2):119-26. 